\title{
DESARROLLO DE UN CUESTIONARIO DE COMPETENCIAS EN TIC PARA PROFESORES DE DISTINTOS NIVELES EDUCATIVOS
}

\section{DEVELOPMENT OF AN ICT COMPETENCES QUESTIONNAIRE FOR TEACHERS ACROSS DIFFERENT EDUCATIONAL LEVELS}

\author{
Dra. J. Carmen Fernández de la Iglesia. \\ c.delaiglesia@usc.es \\ Dra. M. Carmen Fernández Morante. \\ carmen.morante@usc.es \\ Dra. Beatriz Cebreiro López. \\ beatriz.cebreiro@usc.es
}

\begin{abstract}
Universidad de Santiago de Compostela. Facultad de Ciencias de la Educación Departamento de Didáctica y Organización Escolar. Rúa Prof. Vicente Fráiz Andón, s/n. Campus Vida?15782 Santiago de Compostela (España)
\end{abstract}

Actualmente, las competencias para la integración de las TIC en el aula se han convertido en habilidades necesarias para los profesores en su práctica profesional. En este estudio, se ha desarrollado y validado un cuestionario para la evaluación de competencias en TIC del profesorado en diferentes niveles educativos a través de un análisis factorial confirmatorio. Este instrumento abarca aspectos de habilidades técnicas, uso educativo, y diseño de materiales digitales y entornos de aprendizaje. Podría ser útil para investigación en el campo de la tecnología educativa y como punto de partida para diseñar programas de educación continua en TIC para docentes.

Palabras clave: competencias TIC, análisis factorial confirmatorio, cuestionario, profesores.

Actually, the competence to integration ICT in class skills have become in necessary for the teacher in their professional practice. In this research we have developed and validate a questionnaire for to assess the ICT skills of teachers in different educational levels through a confirmatory factorial analysis. This instrument covers technical skills, educational environment and design digital material and learning environments. Could be useful to research in educational technology area and how starting point for design educational continue programs in ICT for the teachers.

Keywords: ICT competences, confirmatory factor analysis, questionnaire, teachers.

Píxel-Bit. Revista de Medios y Educación. No 48 Enero 2016. ISSN: 1133-8482. e-ISSN: 2171-7966. doi: http://dx.doi.org/10.12795/pixelbit.2016.i48.09 


\section{Introducción.}

Las Tecnologías de la Información y la Comunicación (TIC) son consideradas actualmente como indispensables para la participación plena en la Sociedad del Conocimiento (Iniesta, Sánchez \& Schlesinger, 2013; Tondeur, Van \& Valcke, 2007). Este contexto requiere que todos los ciudadanos, especialmente los profesores, adquieran nuevas habilidades personales, sociales y profesionales para afrontar con cambios continuos en todas las áreas.

En las últimas décadas, la mejora de la educación a través de la integración de las TIC en contextos educativos se ha convertido en un tema de gran relevancia para la investigación. Diversos autores (Thompson, Simonson \& Hargrave, 1992; Cabero, 1998; Castaño 1994) desarrollaron desde los años 90 un modelo didáctico-curricular que sitúa las TIC en los procesos educativos entendiendo que éstas por sí mismas no transforman las prácticas y que son sólo un elemento más del proceso de enseñanza por lo que deben ser estudiadas teniendo en cuenta cuatro dimensiones que se relacionan entre sí:

El primero de los aspectos (TIC e infraestructuras disponibles) hace referencia a la dotación existente en los centros desde una doble perspectiva cuantitativa $\mathrm{y}$ cualitativa (cantidad y calidad: de qué medios disponen y en qué condiciones están) y a cómo son adquiridos. En este sentido, desde el comienzo del siglo XXI, las distintas administraciones públicas nacionales han primado el desarrollo de políticas operativas para proporcionar de medios a los centros educativos (Valverde, Garrido \& Sosa, 2010).

En segundo lugar, los aspectos organizativos para la integración de las TIC en los centros educativos, se centra en los elementos organizativos que se consideran necesarios para la incorporación de los medios y tecnologías en los centros educativos, lo que se considera que repercute tanto en la frecuencia de uso de las TIC por parte del profesorado como en el tipo de uso que realicen de las mismas (Cabero, 2004; Tejedor \& García, 2006).

En tercer lugar, los usos que el profesorado hace de las TIC en sus prácticas y las funciones que les atribuyen, aborda las ideas previas del profesorado sobre sus posibilidades didácticas, el uso que hacen de ellas, las funciones que les atribuyen y las principales dificultades que experimentan (Sigalés, Mominó, Meneses \& Badía, 2008).

Finalmente, la formación y competencias en TIC de los profesores, dimensión que supone el objeto de estudio del presente trabajo, se centra en el conocimiento técnico, didáctico y para el diseño de contenidos y medios del profesorado, necesidades formativas percibidas para su integración en las prácticas, modalidades más adecuadas y propuestas para la mejora (Cabero, 2004). Las competencias en TIC de los profesores han sido identificadas repetidamente como un elemento clave para la integración plena de las TIC en la práctica educativa (Almerich, Suárez, Orellana \& Díaz, 2010; Bosco, 2009; Correa \& Martínez, 2010; Ertmer \& Ottenbreif, 2010; Llorente, 2008; Mueller, Wood, Willoughby, Ross \& Specht, 2008; Suárez, Almerich, Gargallo \& Aliaga, 2013). Las competencias en TIC de los profesores pueden ser entendidas como el conjunto de conocimientos y habilidades que un profesor debe poseer para poder usar las herramientas tecnológicas como recursos educativos integrados en la práctica diaria (Suárez, Almerich, Díaz \& Fernández, 2012). Estas 
competencias han sido reconocidas como un factor endógeno que afecta al grado de impacto de las TIC en las escuelas (Losada, Karrera \& Jiménez, 2012).

Desde el punto de vista educativo, el enfoque por competencias implica tanto para el profesorado como para los alumnos, la adquisición de un saber para «actuar», de manera que, se consiga la aplicación de los conocimientos en situaciones prácticas, en contextos reales y concretos. Los docentes deberán conseguir, no solo la adquisición de conocimientos sobre las TIC sino su aplicación en su contexto concreto de aula: la formación basada en competencias necesita que se lleve a cabo una integración de saberes, relacionados con funciones y tareas profesionales concretas que se desarrollen en las situaciones de trabajo (Ruiz, Rubia, Anguita \& Fernández, 2010).

La formación de los profesores debe incluir conocimientos y habilidades que les permitirán dominar recursos tecnológicos e integrarlos de manera exitosa en contextos educativos (Almerich et al, 2010). Instituciones gubernamentales y nogubernamentales de muchos países (Estados Unidos, Colombia, Reino Unido, Chile, Australia, Portugal...) han elaborado guías sobre las competencias en TIC que los profesores (y estudiantes de magisterio) necesitan para su práctica profesional. Se han desarrollado estándares basados en estos modelos de competencias para determinar el conocimiento y las destrezas que un profesor debe poseer para realizar un uso efectivo de las TIC en el proceso de enseñanzaaprendizaje. La utilidad de la definición de estándares en TIC es indudable. Por una parte, permiten a los profesionales saber lo que se espera en cuanto a su función profesional y, en concreto en lo que respecta a la integración de las TIC en su práctica docente. De esta manera, los profesores pueden identificar fácilmente sus necesidades formativas en este ámbito y como consecuencia, definir sus itinerarios formativos a tal efecto. Los distintos estándares elaborados se agrupan en varias dimensiones y cada profesor puede necesitar formarse en una, varias o todas ellas.

Por ello, existe una productiva línea de investigación para identificar los múltiples determinantes para la integración de las TIC en educación (Fernández \& Cebreiro, 2002; Guzman \& Nussbaum, 2009; Koehler \& Mishra, 2009; Markauskaite, 2007; Suárez et al., 2013; Tondeur et al., 2007). Estos factores se han agrupado normalmente en diferentes dimensiones que varían de un estudio a otro, pero que a menudo incluye las dimensiones instrumental/manejo técnico, educativa/ didáctica, evaluativa, comunicacional/ relacional y aspectos personales/ actitudinales (Guzman \& Nussbaum, 2009).

La dimensión instrumental ha sido reconocida como el principal factor que afecta al uso de las TIC. Esta dimensión está relacionada con el manejo técnico de las mismas y definida como el uso apropiado del hardware y el software en un contexto educativo (Guzman \& Nussbaum, 2009). Aunque la dimensión instrumental o tecnológica es básica y necesaria, esta dimensión sola no es suficiente para la integración de las TIC en el aula. Las competencias pedagógicas, englobadas en la denominada dimensión de conocimiento didáctico de los profesores, también son necesarias para incorporar las tecnologías en las actividades educativas. El conocimiento didáctico incluye la habilidad de los profesores para usar las TIC como herramientas para la evaluación del 
aprendizaje de los alumnos y en el proceso de enseñanza-aprendizaje, junto con el uso de herramientas TIC de comunicación con fines educativos, entre profesores, entre profesores y alumnos así como con la familia o el entorno.

Las dimensiones personal o actitudinal se refieren a las actitudes de los profesores hacia la integración y el uso de las TIC en las aulas. Este aspecto se ha convertido en un área de investigación independiente, reflejado en numerosos estudios y en la existencia de instrumentos validados para medir las competencias de esta dimensión (Hernández, Martínez, García, Herrera \& Rodríguez, 2013).

Recientemente, la creación de medios educativos digitales ha ganado relevancia debido a la desaparición de límites claros entre consumidores y productores digitales $(\mathrm{Li}$, 2012). Hoy en día, se espera que los profesores no sean simples consumidores de medios y recursos educativos, sino que también pueda diseñar y crear recursos adaptados a las necesidades de los estudiantes (Cabero, 2004). Esto, a su vez, da lugar a la necesidad de instrumentos que evalúen niveles de competencias en TIC de los profesores de los distintos niveles educativos.

Aunque recientemente se ha realizado una gran cantidad de estudios sobre la formación en TIC de los profesores (Almerich, Suárez, Orellana, Belloch, Bo \& Gastaldo, 2005; Cabero \& Llorente, 2006; Hsu, 2010; Jizat, 2012; Jizat \& McKay, 2011; Markauskaite, 2007; Mueller et al., 2008; Papanastasiou \& Angeli, 2008; Suárez et al., 2013; Tondeur et al., 2007), e incluso se han propuesto y validado modelos para integrar las TIC en contextos educativos (Chai, Ling, Tsai \& Lee Wee, 2011; Hsu, 2010; Markauskaite, 2007; Suárez et al., 2013), no conocemos instrumentos validados que examinen de manera exhaustiva la formación de los profesores para el uso de las TIC en el proceso de enseñanza-aprendizaje. Los modelos existentes no se centran específicamente en estos aspectos sino en contrastar los factores asociados a la integración de las TIC en las aulas, accesibilidad a equipos, usos concreto de las TIC por parte de los profesores o las actitudes de los profesores hacia las TIC. Además, ninguno de esos estudios han validado cuestionarios adaptados para profesores de distintos niveles educativos.

El objetivo de este estudio fue desarrollar y validar un cuestionario para evaluar las competencias en TIC de los profesores de distintos niveles educativos (educación infantil y primaria, educación secundaria, formación profesional), que pudiera ser útil con fines de investigación y como punto de partida para el desarrollo de programas de formación continua para profesores. Para ello, se ha desarrollado un instrumento amplio que cubre aspectos de las TIC incluyendo manejo técnico, uso didáctico y diseño de materiales digitales y entornos digitales de aprendizaje.

\section{Metodología.}

\subsection{Muestra.}

A partir de una población de 32.906 profesores de Educación Infantil y Primaria, Educación Secundaria y Formación Profesional de Galicia, se seleccionó la muestra para esta investigación, que estuvo constituida finalmente por 522 profesores, mediante un procedimiento de muestreo aleatorio simple. El error muestral se estableció en 4,26 (nivel de confianza: 95\%). 
i1 Conectar ordenador y periféricos (impresora, scanner, webcam, etc.)

i2 Instalar Software

i3 Administrar los recursos de un ordenador a través de un Sistema Operativo (p.e.Windows, Linux, Mac, etc.)

i4 Utilizar y gestionar software de seguridad (Antivirus, anti-espías, optimizadores del sistema operativo, etc.)

i5 Elaborar y editar textos en formato digital (utilizando software como p.ej. Word, Writer, World Perfect, etc.)

i6 Elaborar y editar presentaciones en formato digital (utilizando software como p.ej. Power Point, Impress, etc.)

i7 Diseñar y gestionar Hojas de cálculo (utilizando software como p.ej. Excel, Calc, etc.)

i8 Crear y editar imágenes digitales (utilizando software como p.e. Paint NET, Photoshop, Picsizer, etc.)

i9 Cambiar/exportar en diferentes formatos digitales (texto, audio, imagen, vídeo)

Comunicarse por correo electrónico utilizando programas cliente (como p.e. Eudora, Thunderbird, Outlook Express, etc)

Utilizar herramientas de comunicación síncrona vía web (Chat, servicios de mensajería instantánea -tipo

Messenger, Skype, otras herramientas de videoconferencia/audioconferencia, webminars, etc.)

Utilizar herramientas de comunicación asíncrona vía web (foros, listas de distribución, webmail, etc)

i13 Buscar y seleccionar información en Internet

i14 Gestionar y organizar las TIC en el aula para promover su uso en los procesos de enseñanza-aprendizaje

Aplicar diferentes metodologías y estrategias didácticas de uso de las TIC en los procesos de Enseñanzaaprendizaje.

i16 Seleccionar las TIC más adecuadas en función de los objetivos y modelos de enseñanza aplicados

i17 Proponer usos diversificados de las TIC en función de los objetivos y modelos de enseñanza aplicados

i18 Utilizar diferentes TIC para facilitar el autoaprendizaje e individualizar la enseñanza

i19 Diseñar actividades que promuevan el uso de las TIC como recursos para el aprendizaje

i20 Utilizar diferentes TIC para facilitar el acceso por el alumno a más información/otros contextos

i21 Utilizar las TIC para facilitar el recuerdo de la información y reforzar los contenidos

i22 Utilizar las TIC para crear/modificar actitudes en los alumnos

i23 Utilizar diferentes TIC para captar la atención y motivar a los alumnos

i24 Aplicar TIC para desarrollar la creatividad del alumnado

Utilizar diferentes TIC para ofrecer feed-back o retroalimentación al alumno en el proceso de enseñanzaaprendizaje

Utilizar TIC para hacer el seguimiento/supervisar actividades de aprendizaje (trabajos de investigación, actividades de refuerzo educativo, etc.)

Utilizar diferentes TIC para evaluar conocimientos y habilidades de los estudiantes

Aplicar criterios didácticos de calidad en el diseño de contenidos educativos.

i30 Desarrollar una webquest e integrarla en el proceso de enseñanza-aprendizaje.

Utilizar Gestores de contenidos -Content Management Systems: CMS- (como por ejemplo Drupal, Joomla) para crear sitios web dinámicos.

i32 Crear y dinamizar redes y comunidades educativas virtuales

i33 Crear actividades interactivas

i34 Crear tutoriales interactivos

i35 Crear simulaciones y animaciones

i36 Crear Mapas conceptuales interactivos

i37 Crear Material Multimedia educativo

i38 Crear Vídeos didácticos digitales

Desarrollar un weblog temático y promover su utilización en el

proceso de enseñanza-aprendizaje por los alumnos.

i40 Crear un periódico escolar digital

Utilizar software educativo para el diseño de materiales digitales (p.e. CMapTools, JClic, Hot potatoes, Wink,

Exelearning, VTS, Squeak, etc.)

Crear una Wiki y promover su utilización en el proceso de enseñanzaaprendizaje

como medio de expresión, creación colectiva y debate.

i43 Saber integrar contenidos educativos digitales en sistemas de formación y colaboración en red

i44 Crear y gestionar espacios virtuales de enseñanza-aprendizaje y/o colaboración

Ayudar a profesores y alumnos a desarrollar su portafolio digital (estructura de conocimiento y catálogo de competencias)

Tabla 1. Cuestionario de Competencias TIC de los profesores. 
La muestra final está compuesta por profesores con edades comprendidas entre los 24 y los 66 años (media 43, desviación típica 8,57), que podemos dividir en rangos de edad del siguiente modo: $7,7 \%$ entre $20 \mathrm{y}$ 30 años, 29,5\% entre 31 y 40 años, $42,4 \%$ entre 41 y 50 años; 19,3\% entre 51 y 60 años, y $1,2 \%$ entre 61 y 66 años. En cuanto a género, el 45,2\% eran profesores varones. El $14 \%$ de los profesores tenían una experiencia docente entre 1 y 5 años, el 33,5\% entre 6 y 15 años, el $36,6 \%$ entre 16 y 25 años, y el restante $15,9 \%$ tenía más de 25 años de experiencia. Además, dividiendo por niveles educativos, el $26,3 \%$ pertenecían a centros de educación infantil y primaria, el 63,4\% a centros de educación secundaria, y el $10,3 \%$ a profesores de formación profesional.

\subsection{Cuestionario.}

Para la construcción del presente cuestionario, se tuvieron en cuenta las principales referencias teóricas y bibliográficas que fundamentan la investigación de la capacitación del profesorado en el uso de las TIC que podían apuntar los indicadores significativos para dar respuesta al problema de investigación. Es de señalar que también se revisaron diferentes cuestionarios elaborados para otras investigaciones que estaban directa o indirectamente relacionadas con este tema (Cabero, Llorente, \& Puentes, 2008).

Revisada la literatura y los instrumentos realizados para otros estudios previos se formuló una primera versión del instrumento llevado a cabo por jueces expertos en el área de Tecnología Educativa de seis países (Austria, Hungría, Dinamarca, Inglaterra, España y Alemania), que daría lugar a la versión definitiva.
Se realizaron análisis preliminares con 61 ítems, algunos de los cuáles fueron descartados porque: a) fueron considerados reiterativos o poco claros, o b) presentaron pesos inferiores a 0.4 en un análisis factorial exploratorio previo. Así, el cuestionario final contenía un total de 45 ítems (ver Tabla 1) evaluados mediante una escala Likert de 5 puntos (desde «nada competente»a «totalmente competente»).

\subsection{Análisis de datos.}

Siguiendo investigaciones previas (Hernández et al., 2013; Kline, 2011), se llevó a cabo un procedimiento en dos pasos para obtener los análisis de validez de constructo. En primer lugar, se realizó un análisis factorial exploratorio tras comprobar que se cumplían los supuestos de homogeneidad de varianza (test de Bartlett), correlación de las variables (índice KMO index), e idoneidad de la muestra (análisis de la diagonal principal de la matriz de correlaciones anti-imagen) (García, Gil \& Rodríguez, 2000). El análisis exploratorio consistió en un análisis de componentes principales a partir de la matriz de correlaciones. Se empleó el test scree (Cattell, 1966) para tomar la decisión sobre la selección del número de componentes a extraer. Dado que las rotaciones oblicuas han sido recomendadas cuando se espera la existencia de correlación entre factores (Fabrigar, Wegener, MacCallum \& Strahan, 1999), los componentes extraídos se rotaron usando el procedimiento Promax (Hendrickson \& White, 1964), para así facilitar la interpretación de los resultados.

El segundo paso consistió en un análisis factorial confirmatorio utilizando la estimación de la máxima verosimilitud. Puesto que el supuesto de normalidad multivariante 
no se cumplió (curtosis $>5$ ), se realizó el procedimiento «bootstrapping» (1000 muestras, nivel de confianza corregido: 95\%). Finalmente, la invarianza de configuración (ratio $\div$ '/grados de libertad para obtener la bondad de ajuste) y métrica (test $\div^{2}$ por pares) fue evaluada para validar la equivalencia del modelo (o estructura de factores) entre los profesores de los distintos niveles educativos (Educación Infantil y Primaria, Educación Secundaria, Formación Profesional).

Además, se calculó el coeficiente de Cronbach de consistencia interna para determinar la fiabilidad del instrumento. Se obtuvieron los índices de correlación ítemtotal para evaluar la contribución de cada ítem al cuestionario. Finalmente, se obtuvo el índice fiabilidad compuesta para medir la fiabilidad de los factores validados en el análisis factorial confirmatorio.

Todos los análisis fueron llevados a cabo usando IBM SPSS Statistics para Mac OS X, v. 20 e IBM SPSS Amos para Windows, v. 21.

\section{Resultados.}

\subsection{Validez de constructo.}

El cuestionario presentó las condiciones necesarias para la realización de un análisis factorial. El test de Bartlett de esfericidad para medir homogeneidad de varianzas $\left(\dot{\div}^{2}=\right.$ 23050.212; $<<0.001 ;$ grados de libertad $=990$; á $=0.005)$ mostró que las varianzas eran efectivamente homogéneas. El índice KMO (0.979) mostró una correlación muy alta (Kaiser, 1974) de las variables incluidas en el análisis. Los valores de la diagonal principal de la matriz de correlaciones anti-imagen se encontraban en el rango entre 0.955 y 0.989 , confirmando la idoneidad de la muestra.
El análisis factorial exploratorio reveló tres factores (Dimensión de manejo técnico, Dimensión de uso didáctico y Dimensión de diseño de materiales educativos digitales/ entornos de aprendizaje) que explicaban el $67 \%$ de la varianza.

El análisis factorial confirmatorio mostró un ajuste adecuado. Aunque el valor absoluto fue significativo $\left(\div^{2}=1942.25\right.$, grados de libertad $=904, \mathrm{p} \mathrm{d} » 0.001$ ), este resultado era esperado dada la sensibilidad de Chicuadrado a modelos con un gran número de variables y un tamaño muestral grande (Hair, Black, Babin \& Anderson, 2010). La razón $\div 2 /$ grados de libertad para obtener la bondad de ajuste proporcionó un valor aceptable $\left(\div^{2} /\right.$ grados de libertad $=2.149$ ), puesto que valores inferiores a tres pueden ser considerados buenos (Hair et al., 2010). Además, la raíz cuadrada del promedio de la suma de los cuadrados de los residuos (Standardized Root Mean Square Residual, o SRMR) era menor de 0.08 (SRMR $=0.040)$, lo que se considera un buen ajuste $(\mathrm{Hu} \&$ Bentler, 1999). La raíz cuadrada del error de aproximación (Root Mean Square Error of Approximation, o RMSEA) y el valor de probabilidad asociado también indicaron un ajuste adecuado (RMSEA $=0.047, \mathrm{p}=0.960>$ $0.05)$.

En cuanto a los índices de ajuste incrementales, todos los valores mostraron un buen ajuste ( $>0.9$ ). En concreto, el índice de ajuste comparativo (CFI), el índice de ajuste incremental (IFI) y el índice de TuckerLewis (TLI) presentaron valores de 0.954 , 0.950 , y 0.955 , respectivamente, indicando una gran bondad de ajuste (Hair et al., 2010).

Dado que las tres dimensiones evaluaban competencias en TIC de los profesores, no resultó extraño comprobar que el modelo presentaba una alta covarianza entre los 


\begin{tabular}{lllll|ll}
\hline USO TÉCNICO & \multicolumn{2}{l|}{ USO DIDÁCTICO } & \multicolumn{2}{l}{ DISEÑ DE MEDIOS } \\
\hline$I T E M$ & $P E S O$ & \multicolumn{1}{l|}{ ÍTEM } & PESO & ÍTEM & PESO \\
\hline 1 & $0.76^{* *}$ & 14 & $0.82^{* *}$ & 30 & $0.75^{* *}$ \\
2 & $0.78^{* *}$ & 15 & $0.85^{* *}$ & 31 & $0.68^{* *}$ \\
3 & $0.72^{* *}$ & 16 & $0.86^{* *}$ & 32 & $0.82^{* *}$ \\
4 & $0.77^{* *}$ & 17 & $0.87^{* *}$ & 33 & $0.85^{* *}$ \\
5 & $0.69^{* *}$ & 18 & $0.85^{* *}$ & 34 & $0.88^{* *}$ \\
6 & $0.77^{* *}$ & 19 & $0.86^{* *}$ & 35 & $0.77^{* *}$ \\
7 & $0.66^{* *}$ & 20 & $0.83^{* * *}$ & 36 & $0.83^{* *}$ \\
8 & $0.72^{* *}$ & 21 & $0.82^{* *}$ & 37 & $0.82^{* *}$ \\
9 & $0.81^{* *}$ & 22 & $0.75^{* *}$ & 38 & $0.78^{* *}$ \\
10 & $0.62^{* *}$ & 23 & $0.79^{* *}$ & 39 & $0.81^{* *}$ \\
11 & $0.69^{* *}$ & 24 & $0.79^{* *}$ & 40 & $0.82^{* *}$ \\
12 & $0.73^{* *}$ & 25 & $0.85^{* *}$ & 41 & $0.80^{* *}$ \\
13 & $0.66^{* *}$ & 26 & $0.85^{* *}$ & 42 & $0.85^{* *}$ \\
& & 27 & $0.80^{* *}$ & 43 & $0.88^{* *}$ \\
& & 28 & $0.77^{* *}$ & 44 & $0.87^{* *}$ \\
\hline
\end{tabular}

$* * * \mathrm{pd} \gg 0.001, * * \mathrm{pd} \gg 0.01$

Tabla 2. Estimación de parámetros para cada ítem en las dimensiones del análisis factorial confirmatorio.

factores (entre 0.79 y 0.82 ). En consecuencia, otro análisis factorial confirmatorio fue llevado a cabo con un solo factor, que no mostró un ajuste adecuado $\left(\div^{2} /\right.$ grados de libertad = 4.026). Así, se concluyó que el modelo de tresque el modelo de 3 factores parece más apropiado para evaluar las competencias en TIC de los profesores.

Todos los ítems mostraron una adecuada contribución de la dimensión a la que pertenecen, con pesos entre 0.62 y 0.88 (ver Tabla 2).

Finalmente, puesto que el cuestionario se dirige a profesores de distintos niveles educativos (Educación Infantil y Primaria, Educación Secundaria, Formación Profesional), se han examinado la invarianza métrica y de configuración para asegurar la validez del cuestionario en los tres niveles educativos. Se obtuvo un ajuste adecuado $(<3)$ cuando los tres grupos fueron incluidos en el modelo $\left(\div^{2} /\right.$ grados de libertad $\left.=1.877\right)$. Además, las pruebas $\div^{2}$ entre pares de grupos revelaron que en al menos un ítem no se observaron diferencias significativas. Por ello, podemos concluir que este cuestionario es una herramienta válida para evaluar las competencias en TIC en profesores de los distintos niveles educativos.

\subsection{Análisis de fiabilidad.}

El análisis de consistencia interna mostró un coeficiente alfa de Cronbach muy alto (á= 0.981 ), indicando que el cuestionario presenta una alta consistencia interna (DeVellis, 2003). 
Todos los ítems propuestos en la escala presentaron un índice de discriminación alto, entre 0.563 y 0.831 . Basándonos en estos resultados, y en el hecho de que no se obtendrían mejoras en la consistencia interna por la eliminación de ninguno de los ítems, se mantuvieron todos los ítems en el cuestionario.

Además, el índice de fiabilidad compuesta también proporcionan valores adecuados $(>0.7)$. Concretamente, el índice de fiabilidad compuesta fue 0.93 para la dimensión de manejo técnico, y 0.97 para las dimensiones de uso didáctico y de diseño de materiales educativos digitales/entornos de aprendizaje.

En conjunto, podemos afirmar que el presente cuestionario es una herramienta fiable.

\section{Discusión.}

Aunque existen estudios previos que han propuesto y validado modelos de integración de las TIC en contextos educativos (Chai et al., 2011; Hsu, 2010; Markauskaite, 2007; Suárez et al., 2013), no conocemos instrumentos validados que evalúen las competencias en TIC de profesores de distintos niveles educativos y sus necesidades de formación. Cuestionarios utilizados en estudios previos incluyen aspectos comunes, pero existe una gran heterogeneidad en los ítems evaluados. Además, no existe ninguna herramienta que haya sido validada para poder ser utilizada en profesores de distintos niveles educativos. Dada la heterogeneidad en la formación y características de la población de profesores de distintos niveles educativos, este aspecto es de gran relevancia, puesto que permite determinar hasta qué punto se pueden generalizar los resultados de un cuestionario entre distintos grupos (Hair et al., 2010). El objetivo del presente trabajo fue, por tanto, desarrollar y validar una herramienta que incorporaba dimensiones no incluidas en cuestionarios previos, para poder evaluar de manera exhaustiva las competencias en TIC de los profesores de distintos niveles educativos.

El análisis exploratorio indica que los ítems del cuestionario pueden ser agrupados en tres dimensiones básicas, que se corresponden con dimensiones identificadas en estudios previos (Hsu, 2010; Markauskaite, 2007; Suárez, Almerich, Gargallo \& Aliaga, 2010; Suárez et al., 2012; Tondeur et al., 2007): la dimensión instrumental, la dimensión didáctica y la dimensión de diseño de medios y entornos de aprendizaje digitales. El análisis factorial confirmatorio mostró valores de ajuste adecuados, a pesar del número de ítems del cuestionario. Además, se confirmó que el uso de tres dimensiones presentaba un mejor ajuste que el uso de una sola dimensión (competencias en TIC).

Así, los análisis realizados en este trabajo confirman el carácter multidimensional de las competencias TIC del profesorado, ya señalado en estudios previos (Cabero, 2004; Guzman \& Nussbaum, 2009). Atender a esta heterogeneidad es de suma importancia para una integración plena de las tecnologías en el aula, tal como se ha recogido en distintos estándares de necesidades de formación del profesorado en distintos países.

La dimensión técnica es un área clave que ha sido señalada en prácticamente todos los estudios relativos a las competencias en TIC de los profesores, puesto que el manejo técnico de los recursos TIC es necesario para su aplicación en contextos educativos. 
La dimensión didáctica o pedagógica también ha sido incluida en los modelos sobre integración de las TIC en la educación (Koehler \& Mishra, 2009; Markauskaite, 2007; Suárez et al., 2013). Los ítems referidos a esta dimensión en el cuestionario del presente estudio incluían aspectos relacionados con la evaluación, la comunicación y el uso de las TIC con propósitos educativos. Estudios previos (Guzman \& Nussbam, 2009; Hsu, 2010; Jizat, 2012; Jizat \& McKay, 2011) señalaron la evaluación y la comunicación mediante TIC como dimensiones separadas. Sin embargo, el análisis exploratorio del presente estudio apuntó a que esos aspectos pueden estar integrados en una misma dimensión.

El cuestionario validado en este estudio incluye una evaluación de las competencias de los profesores para diseñar sus propios recursos digitales de aprendizaje. Estudios recientes han descrito las ventajas de crear materiales adaptados a las características de cada aula (Cabero, 2004; Li, 2012). La capacidad de los profesores para crear y modificar recursos digitales posibilita la adaptación de la experiencia de aprendizaje a distintos ritmos, lo que constituye uno de los mayores beneficios potenciales del uso de las TIC en las aulas (Çelik, Arkýn \& Sabriler, 2012; McLoughlin \& Lee, 2010; Tarik \& Karim, 2012). La última generación de herramientas TIC ha simplificado la creación de recursos y medios digitales, permitiendo que los profesores mejoren y personalicen los materiales para el proceso de aprendizaje en el aula sin necesidad de competencias propias de los programadores informáticos (Peters \& Slotta, 2010; Thompson, 2007).

Es por ello de gran importancia contemplar aspectos técnicos, didácticos y de diseño de medios y materiales en la formación de los profesores. Tal como se ha señalado que las políticas operativas son necesarias pero insuficientes para la integración de las TIC en el aula (Valverde et al., 2010), la formación de los profesores, ya sea en grados, másters profesionalizantes o cursos de formación continua deben contemplar la importancia no solo de aspectos básicos de uso de las tecnologías, sino de las competencias pedagógicas necesarias para no limitarse a utilizar nuevas herramientas como instrumentos transmisores de información y como fuente de motivación extrínseca para los estudiantes para metodologías tradicionales de enseñanza (Sigalés et al., 2008).

La principal ventaja de este cuestionario reside en la posibilidad de su aplicación en dos áreas diferenciadas. Primero, este cuestionario de 45 ítems proporciona información cualitativa extensa sobre las competencias adquiridas por los profesores. Del mismo modo, puede ser utilizado para recabar información de las necesidades de formación para poblaciones específicas de profesores. Se puede argumentar que la lista de ítems podría ser más amplia (de hecho, como se señaló en el método se realizaron análisis preliminares con un mayor número de ítems), pero los análisis han demostrado que estos ítems son fiables y válidos para el estudio de las distintas dimensiones de competencias relevantes para la integración de las TIC en el aula.

En segundo lugar, dada la bondad de ajuste mostrada por el análisis factorial confirmatorio, esta herramienta parece ser apropiada para la investigación sobre competencias en TIC de los profesores. Como ventaja adicional, se ha contrastado la validez de este cuestionario para su uso en tres niveles educativos: Educación Infantil y 
Primaria, Educación Secundaria y Formación Profesional. Este es, a nuestro entender, el primer estudio en el que se obtienen medidas de invarianza métrica y de configuración con este fin, lo que suponen un requisito para la aplicación de un modelo y/o escala de medida de grupos heterogéneos como son los profesores de distintos niveles educativos. La importancia de estos análisis residen en el uso potencial del cuestionario para comparar el nivel de competencias entre grupos de profesores de distintos niveles educativos. Los resultados mostraron que el modelo de tres dimensiones parece ser válido para evaluar y comparar las competencias en TIC de los profesores en esos tres niveles educativos.

Estudios futuros podrían incluir otros niveles educativos, como profesores universitarios. Además, el modelo podría ser aumentado y aplicado en la comparación de competencias en TIC de profesores de distintos países. Además, esta herramienta podría ser utilizada conjuntamente con otras herramientas validadas (por ejemplo, de actitudes de los profesores; ver Hernández et al., 2013) para desarrollar modelos más complejos para la evaluación de la integración de las TIC en entornos educativos.

\section{Fuentes de financiación.}

Este trabajo forma parte del proyecto 141882-LLP1-2008-1-AT-COMENIUS-CMP, denominado «ICTeachers» financiado por la Unión Europea a través del programa UE Lifelong learning programme.

\section{Referencias bibliográficas.}

Almerich, G., Suárez, J. M., Orellana, N., Belloch, C., Bo, R. \& Galtaldo, I. (2005).
Diferencias en los conocimientos de los recursos tecnológicos en profesores a partir del género, edad y tipo de centro. Revista Electrónica de Evaluación e Investigación Educativa, 11(2), 127-146.

Almerich, G., Suárez, J.M., Orellana, N. \& Díaz, M.I. (2010). La relación entre la integración de las tecnologías de la información y comunicación y su conocimiento. Revista de Investigación Educativa, 28 (1), 31-50.

Bosco, A. (2009). Las tecnologías de la información y la comunicación en la formación del profesorado: Lineamientos, actualidad y prospectiva. Razón y Palabra, 63. Recuperado de http:// www.razonypalabra.org.mx/n63/abosco.html

Cabero, J. (1998). La formación del profesorado en medios audiovisuales. $E l$ siglo que viene, 4-5, 14-19.

Cabero, J. (2004). Formación del profesorado en TIC: el gran caballo de batalla. Comunicación y Pedagogía. Tecnologías y Recursos Didácticos, 195, 27-37.

Cabero, J. \& Llorente, M. C. (2006). La rosa de los vientos. Dominios tecnológicos de las TICs por los estudiantes. Sevilla: Grupo de Investigación Didáctica.

Cabero, J., Llorente, M. C. \& Puentes, A. (2008). Alfabetización digital: Un estudio en la pontificia católica madre y maestra. Sevilla: Fortic.

Castaño, C. (1994). Análisis y evaluación de las actitudes de los profesores hacia los medios de enseñanza. Bilbao: Universidad del País Vasco.

Cattell, R. B. (1966). The scree test for the number of factors. Multivariate behavioral research, 1(2), 245-276. doi: 10.1207/ s15327906mbr0102_10

Çelik, S., Arkýn, E. \& Sabriler, D. (2012). EFL Learners' Use of ICT for Self-Regulated 
Learning. Journal of Language \& Linguistics Studies, 8(2), 98-118.

Chai, C. S., Ling, J. H., Tsai, C. C. \& Lee, L. (2011). Modeling primary school pre-service teachers' Technological Pedagogical Content Knowledge (TPACK) for meaningful learning with information and communication technology (ICT). Computers \& Education, 57 (1), 1184-1193. doi: 10.1016/ j.compedu.2011.01.007

Correa, J. M. \& Martínez, A. (2010). ¿Qué hacen las escuelas innovadoras con la tecnología?: Las TIC al servicio de la escuela y la comunidad en el colegio amara berri. Teoría de la Educación. Educación y Cultura en la Sociedad de la Información, 11, 230261.

DeVellis, D. R. F. (2003). Scale development: theory and applications. Sage Publications: USA.

Ertmer, P. A. \& Ottenbreit, A.T. (2010). Teacher tecnology change; How knowledge, confidence, beliefs and culture intersect. Journal of Research on Tecnology in Education, 42 (3), 255-284.

Fabrigar, L. R., Wegener, D. T., MacCallum, R. C. \& Strahan, E. J. (1999). Evaluating the use of exploratory factor analysis in psychological research. Psychological methods, 4 (3), 272-299. doi: http://dx.doi.org/ 10.1037/1082-989X.4.3.272

Fernández, M. C. \& Cebreiro, B. (2002). La preparación de los profesores para el dominio técnico, didáctico y el diseño/producción de medios en galicia. Innovación Educativa, 12, 109-122.

García, E., Gil, J. \& Rodríguez, G. (2000). Análisis factorial. Madrid: La Muralla.

Guzman, A. \& Nussbaum, M. (2009). Teaching competencies for technology integration in the classroom. Journal of
Computer Assisted Learning, 25 (5), 453-469. doi: 10.1111/j.1365-2729.2009.00322.x

Hair, J. F., Black, W. C., Babin, B. J. \& Anderson, R. E. (2010). Multivariate data analysis (7th Ed.). Englewood Cliffs: Prentice Hall.

Hendrickson, A. E. \& White, P. O. (1964). Promax: A quick method for rotation to oblique simple structure. British Journal of Statistical Psychology, 17 (1), 65-70. doi: 10.1111/j.2044-8317.1964.tb00244.x

Hernández, J. P., Martínez, F., García, F. J., Herrera, M. E. \& Rodríguez, M. J. (2013). Teachers' attitude regarding the use of ICT. A factor reliability and validity study. Computers in Human Behavior, 31, 509-516. doi: 10.1016/j.chb.2013.04.039

Hsu, S. (2010). Developing a scale for teacher integration of information and communication technology in grades 1-9. Journal of Computer Assisted Learning, 26 (3), 175-189. doi: 10.1111/j.13652729.2010.00348.x

Hu, L. T. \& Bentler, P. M. (1999). Cutoff criteria for fit indexes in covariance structure analysis: Conventional criteria versus new alternatives. Structural Equation Modeling: A Multidisciplinary Journal, 6 (1), 1-55. doi: 10.1080/10705519909540118

Iniesta, M. A., Sánchez, R. \& Schlesinger, W. (2013). Investigating factors that influence on ICT usage in higher education: a descriptive analysis. International Review on Public Nonprofit Marketing, 10 (2), 163-174. doi: 10.1007/s12208-013-0095-7

Jizat, J. E. M. (2012). Investigating ICTliteracy assessment tools: Developing and validating a new assessment instrument for trainee teachers in Malaysia (Doctoral dissertation) RMIT University. Retrieved from http://researchbank.rmit.edu.au/eserv/ rmit:160385/Mat_Jizat.pdf 
Jizat, J. E. M. \& McKay, E. (2011). Developing an Instrument of Assessment for ICT Literacy for Trainee Teachers: Preliminary Findings. International Journal of Computer Information Systems and Industrial Management Applications, 3, 552-559.

Kaiser, H. F. (1974). An index of factorial simplicity. Psychometrika, 39 (1), 31-36. doi: 10.1007/BF02291575

Kline, R. B. (2011). Principles and practice of structural equation modeling. New York: Guilford Press.

Koehler, M. J. \& Mishra, P. (2009). What is Technological Pedagogical Content Knowledge?. Contemporary Issues in Technology and Teacher Education, 9 (1), 60-70.

Li, Q. (2012). Undeerstanding enactivism: a study of affordances and constraints of engaging practicing teachers as digital game designers. Education Technology Research and Development, 60 (5), 785-806. doi: 10.1007/s11423-012-9255-4.

Llorente, M. C. (2008). Aspectos fundamentales de la formación del profesorado en TIC. Pixel-Bit, Revista de Medios y Educación, 31, 121- 130.

Losada, D., Karrera, I. \& Jiménez, E. (2012). Factors facilitating successful Educational innovation with ICT in schools. Revista de Psicodidáctica, 17 (1), 113-134. doi: http:// dx.doi.org/10.1387/RevPsicodidact.2243

Markauskaite, L. (2007). Exploring the estructure of trainee teacher's ICT literacy: the main components of, and relationships between, general cognitive and technical capabilities. Educational Technology, Research and Development, 55 (6), 547-572. doi: 10.1007/s11423-007-9043-8

McLoughlin, C. \& Lee, M. J. (2010). Personalised and self regulated learning in the Web 2.0 era: International exemplars of innovative pedagogy using social software. Australasian Journal of Educational Technology, 26 (1), 28-43.

Mueller, J., Wood, E., Willoughby, T., Ross, C. \& Specht, J. (2008). Identifying discriminating variables between teachers who fully integrate computers and teachers with limited integration. Computers \& Education, 51 (4), 1523-1537. doi: 10.1016/ j.compedu.2008.02.003

Papanastasiou, E. C. \& Angeli, C. (2008). Evaluating the use of ICT in Education: Psychometric properties of the survey of factors affecting teachers teaching with tecnology (SFA-T3). Educational Technology \& Society, 11 (1), 69-86.

Peters, V. L. \& Slotta, J. D. (2010). Scaffolding knowledge communities in the classroom: New opportunities in the Web 2.0 era. In M. Jacobson \& P. Reimann (Eds.). Designs for learning environments of the future (pp. 205-232). New York: Springer.

Ruiz, I., Rubia, B., Anguita, R. \& Fernández, E. (2010). Formar al profesorado inicialmente en habilidades y competencias en TIC: perfiles de una experiencia colaborativa. Revista de Educación, 352, 149-178.

Sigalés, C., Mominó, J. M., Meneses, J. \& Badia, A. (2008). La integración de internet en la educación escolar española: Situación actual y perspectivas de futuro. Barcelona: Publicacions a Internet, Direcció de Comunicacio, Universitat Oberta de Catalunya. Recuperado de http:// w w w. u o c . e d u / i n $3 /$ integracion_internet_educacion_escolar/ esp/pdf/informe_escuelas.pdf

Suárez, J., Almerich, G., Gargallo, B. \& Aliaga, F. M. (2010). Las competencias en TIC del profesorado y su relación con el uso de los recursos tecnológicos. Education Policy Analysis Archives/Archivos Analíticos de 
Políticas Educativas, 18 (8). Retrieved from http://epaa.asu.edu/ojs/article/viewFile/755/ 832

Suárez, J. M., Almerich, G., Díaz, I. \& Fernández, R. (2012). Competencias del profesorado en las TIC: Influencia de factores personales y contextúales. Universitas Psychologica, 11 (1), 293-309.

Suárez, J., Almerich, G., Gargallo, B. \& Aliaga, F. M. (2013). Las competencias del profesorado en TIC: estructura básica. Educación XXI, 16 (1), 39-62. doi: 10.5944/ educXX1.16.1.716

Tarik, M. \& Karim, A. (2012). The use of web 2.0 innovations on education and training. Education, 2 (5), 183-187. doi: 10.5923/j.edu.20120205.11

Tejedor, J. \& García, A. (2006). Competencias de los profesores para el uso de las TICs en la enseñanza. Análisis de sus conocimientos y actitudes. Revista Española de Pedagogía, 233, 21-44.

Thompson, A. D., Simonson, M. R. \& Hargrave, C. P. (1992). Educational Technology: A review of the research. Washington: Association for Educational Communications and Technology

Thompson, J. (2007). Is Education 1.0 ready for Web 2.0 students?. Innovate Journal of Online Education, 3(4). Recuperado de http:/ / w w w. i n n o vate o n lin e. in fo/ index.php?view $=$ article $\&$ id $=393$

Tondeur, J., Van, J. \& Valcke, M. (2007). Curricula and the use of ICT in education: Two worlds apart?. British Journal of Educational Technology, 38 (6), 962-976. doi: 10.1111/j.1467-8535.2006.00680.x
Valverde, J., Garrido, M. C. \& Sosa, M. J. (2010). Políticas educativas para la integración de las TIC en Extremadura y sus efectos sobre la innovación didáctica y el proceso enseñanza-aprendizaje: la percepción del profesorado. Revista de educación, 352, 99. 124.
Fecha de recepción: 27-04-2015

Fecha de evaluación: 15-05-2015

Fecha de aceptación: 30-06-2015 